\title{
BONDING BASED TECHNIQUE FOR MESSAGE FORWARDING IN SOCIAL OPPORTUNISTIC NETWORK
}

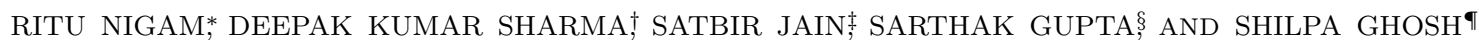

\begin{abstract}
Integrating social networks properties such as centrality, tie strength, etc. into message forwarding protocols in opportunistic networks has grown into a vital major benchmark. The opportunistic network is a demanding network with no set route to travel a message from the source to the destination. During these networks, nodes use possibilities gained based on storecarry-forward patterns to forward communications. Every node that obtains a message when it encounters another node makes selection concerning the forwarding or not necessarily transmitting the message came across. Most of these message forwarding protocols use the benefit of social properties information like contact information and social relationship enclosed by the nodes in the Social Opportunistic Network. In this paper, a Bonding based forwarding technique proposes which is finding direct and indirect bonding among nodes by exploiting contact information and social pattern. In the proposed protocol, we also focus on indirect bonding by finding weakest direct bonded nodes and then replace it with strong indirect bonded nodes of the network. In this work, the balance between transmission delay and network traffic consider by using shortest path map based mobility model. ONE simulator use for simulation and performance of the proposed protocol compare contrary popular approaches for instance Epidemic, PRoPHET, BubbleRap, and Interaction based when using the shortest path map based mobility model. The Bonding based forwarding technique performs adequately well concerning the number of messages delivered, overhead ratio, message dropping and average latency.
\end{abstract}

Key words: Direct and Indirect Bonding based forwarding, Social opportunistic network, ONE Simulator.

AMS subject classifications. $68 \mathrm{M} 12$

1. Introduction. In this digital era, many wireless devices like mobile devices, laptops are available which are using wireless technology for communication. It includes ad-hoc scenario; no infrastructure is present, no topology is used for communication between mobile devices. The situation as mentioned above leads to a random network called opportunistic network where a message is traveled from source to destination by using store-carry-forward pattern. In the opportunistic network environment; no fixed route is available, as the nodes come into the contact of other neighbor nodes, it establishes a random path dynamically to forward a message. Many routing techniques are present for communication in the opportunistic network. Different approaches to map the word in the opportunistic environment are available like diffusion based and context based. Routing protocols which follow diffusion technique, circulate multiple replicas of the message to one-hop neighbor nodes. By distributing, numerous reproductions of the text to nodes increment the possibility of reaching the word to the target node in a speedy manner but raises network traffic. Routing based on context uses information that is the frequency of the node to meet other nodes, distance, speed, location history, etc. to minimize the network traffic but increases transmission delay. As of now, universal knowledge of network topology is the base for very efficient routing and forwarding decisions. Aggregating and swapping the information of network topology in the opportunistic network is inconvenient because of their infrequent connectivity and uncertain mobility. Routing strategies for this type of systems generally build on limited knowledge and on forecasting of future contacts which produces deteriorated routing performance. As the nodes in the opportunistic network encounter periodically, there is a requirement of estimating the quality of links between all combinations of nodes to find out the feasible forwarding option between nodes. Later, a distinct weight assigns to each connection of the nodes by condensing the appropriate encounter information. Then, a neighboring graph of nodes forms for forwarding the messages.

\footnotetext{
*Division of Computer Engineering, University of Delhi, Netaji Subhas Institute of Technology Delhi, India (ritu. nigam2106@gmail.com).

${ }^{\dagger}$ Division of Information Technology, University of Delhi, Netaji Subhas Institute of Technology Delhi, India (dk. sharma1982@yahoo.com), corresponding author.

${ }_{\ddagger}^{\ddagger}$ Division of Computer Engineering, University of Delhi, Netaji Subhas Institute of Technology Delhi, India (jain satbir@yahoo.com).

$\S$ Division of Information Technology, University of Delhi, Netaji Subhas Institute of Technology Delhi, India (sarthak gupta259@gmail.com ).

IDivision of Information Technology, University of Delhi, Netaji Subhas Institute of Technology Delhi, India (ghosh shilpa01@gmail.com).
} 
With the transformation of Online Social Network applications and platforms like Facebook, Twitter, or Linkedin, information about the social intercommunication of nodes has to turn into readily available [1]. Moreover, although the intercontact information in the opportunistic network is varying dynamically, the nodes and the links of a social network stay comparatively stable. Earlier, several approaches, including interaction based [2], communities, similarity, centrality, encounter frequency, total or average contact period and average separation period [3] were adopted to quantify the quality of connections between couples of nodes. Despite, all the approaches as mentioned above have a few shortcomings in the exact depiction of the sending option between nodes.

The leading carrier of mobile devices are human beings, and humans are social by character. These social characteristics play an essential role in forwarding mechanism of the opportunistic social network. Community and centrality of nodes are social characteristics of nodes in the opportunistic social network. With the help of these social characteristics mobility pattern, primary contact nodes, etc. can be identified to predict the path towards the destination. Therefore, it seems that cultural properties are the performance booster elements in the system.

This paper exploits the social network attributes of the opportunistic social network and presents a Bonding based forwarding technique (BBFT) for information propagation. Social relations between nodes evaluate by defining bonding concerning their behavior. The bonding property of the node calculates as a bonding metric determines their concerning behavior. Later, a distinct weight assigns to each connection of the nodes by condensing the appropriate encounter information. Then, a neighboring graph of nodes forms for forwarding the messages behavior. A new bonding metric is specified to measure different forms of bonding behavior regarding contact information and social pattern two types of bonding defined in this paper, i.e., direct bonding and indirect bonding.

The highlights of this work outline as follows:

1. This work inspects the social meeting patterns from the temporal prospects. The explanations of average detachment time and variance introduce the nodes average detachment period as well as the deviation a certain period both by considering it.

2. It inspects that, nodes temporal, social meeting patterns mirror a strong interrelationship. To include this information, two methods design which is named as direct bonding and indirect bonding to forward messages.

3. Based on the direct bonding and indirect bonding methods, an efficient message forwarding technique is proposed to enhance the capability of message forwarding in the opportunistic social network.

4. Through extensive simulations, it is shown that the proposed algorithm is significantly superior to current message forwarding techniques concerning message delivery ratio, dropped messages, overhead, and average latency.

The rest of the paper systematize as follows. Segment 2 presents an overview of previously done research work related to routing protocols. Segment 3 gives the motivation for building the forwarding technique. Segment 4 illustrates the detailed design of the BBFT routing protocol. Segment 5 shows the BBFT algorithm. Segment 6 describes the simulation model using ONE simulator and results. Segment 7 concludes the presented algorithm BBFT and highlights the future work.

2. Literature Review. Research related to forwarding techniques in the opportunistic network done from past decades. Here an overview of these routing techniques is discussed.

Y. Li and R. Bartos, proposed Interaction based Routing Algorithm [2] which utilizes interaction as a twoway effect among nodes like meetings and talking in the social network. It contains two type of components which is direct effects and indirect effects. The immediate effort is considered as a direct impact of a node on another when not disseminated via a third node. The indirect implications regard as an indirect impact of a node on another that propagates through a third node. This algorithm exploits these effects for calculating most popular nodes in the network. F. Li and J. Wu proposed LocalCom [3] protocol which exploits the separation time of nodes to create similarity metrics between every node pair. This local information is then utilized to form communities. The message is forwarded to the destination by gateway nodes in the communities. To develop community structure in this scheme an extended clique clustering used which utilizes virtual links of the network these built communities equipped with specific desirable properties like robust community connection 
and controllable diameter, which can provide intra-community communication based on the single-copy source routing. A diffusion-based routing [4], proposed by Vahdat and Becker is epidemic routing. The message is diffused like a virus in the network to deliver to the destination randomly. This routing strategy works on each host that keeps up a buffer containing texts that it has produced as well as words that it is accumulating for some other hosts. A summary vector is maintained by each node which includes saved messages in its buffer space. This protocol suffers from high network traffic.

A probability-based routing [5], proposed by Lindgren et al. is called PRoPHET. In this routing technique probability of a node to send a message to the destination is calculated by its encounter history with other nodes. This protocol also applies transitivity to get predicted next hop. Every node calculates a matrix called delivery predictability before sending the message to the next node or destination. Based on goggles page rank algorithm, A. Mtibaa et al. proposed people rank [6] protocol. In this protocol, the social properties of a social network are used to classify the nodes. A node with higher ranking uses as a forwarder between two nodes. Ranking of nodes is updated whenever nodes make contact. Daly, E. H., et al. proposed SimBet [7] protocol which uses the concept of ego network. A utility function derived from betweenness centrality and the similarity of the node is used to choose the forwarding nodes. Hui, P. et al. proposed BubbleRap [8] forwarding protocol which applies a community-based approach. Each node has assigned itself global rank and local rank for forwarding purpose. Both positions calculated by using betweenness centrality. By using universal ranking, the message transmitted with the help of hierarchical ranking tree mechanism. Later when the message reached to destination node community than the local ranking of nodes of that community used for forwarding. Kclique used as a community detection strategy in this algorithm. Spyropoulos, T. et al. proposed Spray and Wait protocol [9]. This protocol limits the random dissemination of the message. A fixed amount of message copies are spread to other nodes to reach the destination. Jiho Park et al. proposed ABCON [10] message forwarding protocol by using Artificial bee colony approach. This nature-inspired algorithm mostly prefers for multidimensional and multimodal optimization problems. But the author slightly alters the behavior of the bees by defining their responses as follows. If a bee is carrying no message and searching for a message is known as Scout bee. If a bee is taking a single message with it is called Employed bee. An employed bee always forward that carried a message to Onlooker Bee. Percolation centrality of bee nodes is calculated to forward the message to the destination node in the Social Opportunistic Network. Q. Li et al. proposed selfish node routing [11] in delay tolerant network. A selfish node plays maliciously in the forwarding process by not sending the message to next hop. Sanjay K. Dhurandher et al. proposed HBPR [12] which keep track on movement history of the network nodes to predict next hop of the node. The space of the network partition into identical size cells and a cell allocate to each node as its home location which is familiar to other nodes in the system. The parameters used to transmit the data packets to their destination are the speed of the node with which it is moving and the direction of its movement in the network space by using these parameters of the node, a utility metric measure which shows the appropriateness of a node to deliver the message to the destination. Nodes carrying higher utility value than a defined threshold will become the next forwarder of the word. This protocol also includes a method which acknowledges the received messages to help in the buffer management of the intermediate nodes. Research in OppNets has even started focusing on tackling security issues [13, 14] energy efficiency $[15,16]$ and nature-inspired routing techniques $[17,18]$.

This paper is presenting a Bonding based forwarding technique, for routing, which effortlessly detects the important nodes using bonding information and exploits the indirect connections to raise the forwarding efficiency. To operate the bonding relationship of the Social Opportunistic Network (SON), a metric called bonding metric is illustrated to represent the neighboring relationship of nodes in the beginning. In the Social Opportunistic Network, nodes mostly have the insight of their connections, also called history of frequency of encounter, which encloses both geographical and temporal information. In Bonding based forwarding technique, we choose the states of the average detachment period to abbreviate nodes insight into a metric that is needed. The average detachment duration calculates for a node based on the encounter frequency and duration of their connections in the vicinity of its neighbors. Indeed, a shorter detachment duration emulates a proximate relationship. Direct bonding metric and indirect bonding metric together depicts a unique way to forward a message using a bonding based graph structure. 
3. Motivation. The domain of Social Opportunistic Network (SON) is rising as a distinct paradigm to utilize the social properties of network nodes for designing networking solutions. In recent years, several analysis efforts associated with mobile devices are created to explore the potential of mobile devices. In opportunistic networks, communication is established among mobile devices through routing protocols by using frequent disconnections and uncertain links. According to the Researchers, mobile devices are sharing a close relationship with human society because human beings and vehicles generally carry these devices. Humans always maintain the various types of relationships among themselves called social properties. For example, human beings are usually friendly, and humans with identical behavior mostly devote a long time with each other, being more inclined to share data and resources. Moreover, social properties are crucial to find the nodes mobility pattern and forecast contact opportunities more precisely. Therefore, the SON is capable of determining more positive nodes by using the advantage of the social properties of network nodes.

In literature, many Community-Based and Community-Independent routing protocols have suggested for SON. The Community-Based routing approaches forms communities in the network by taking nodes of common interest. Although, detection and formation of the distributed community are still a difficult task by cause of the dynamic topology changes and complications in data exchange and computation. Due to the difficulty in community detection of these protocols jolts us to adopt Community-Independent routing to select next hop. Besides, the Community-Independent schemes have proposed previously like PROPHET, HiBOP, SimBet, SimBetTS, and People Rank exploit the nodes context information and social properties to send each message to the destination. It reduces the network overhead. As a result, routing protocols following community-independent strategy sidestep the uncovering of community and take on the information of context, social properties (like centrality, tie strength, and similarity), and social meeting pattern to forecast the best forwarder. The implementation of these routing protocols is understandable and manageable. The functioning of Bonding based forwarding technique has been defined to take advantage of Community-Independent routing, to gain a high delivery probability in association with reducing the network overhead, dropped messages and average latency.

4. Proposed Approach. This paper applies the idea to use social information that is more stable and then augment partial intercontact details to implement the efficient message routing protocol in the Social Opportunistic Network. The inspiration behind this proposed idea is that human interaction is a social activity and this social connectedness of common nodes provides a better way to forward a message to any specified destination. Adopting social interaction among people for sending messages in a network has previously been showed to cut down many message replicas at the same time growing the possibility of reaching a letter to its destination.

4.1. Dynamic Weighted Graph. The bonding based forwarding technique, which reveals and employs the essential social bonding to speed up message forwarding in the Social Opportunistic Network, has three steps: Direct bonding, indirect bonding, and message forwarding. A Social Opportunistic Network is considered as a dynamic weighted graph which can characterize as a time progression of network graph denoted by $\left(G_{s 1}\right.$, $\left.G_{s 2}, G_{s 3}, \ldots \ldots \ldots, G_{s t}, \ldots.\right)$ where $G_{s t}=\left(V_{s t}, E_{s t}, F_{s t}, W_{s t}\right)$ represents the state of the SON at time t. In this social graph, $V_{s t}$ represents nodes, $E_{s t}$ represents links between nodes, $W_{s t}=w_{a b}^{t}, \in[0,1) \mid \mathrm{a}, \mathrm{b} \in V_{s t}$ and (a, b) $\in E_{s t}$ depicts the bunch of weights on edges at time t, and $F_{s t}: E_{s t} \rightarrow W_{s t}$ is a function that credits weights to links. The pair of node group and link group modify over time. A link $(\mathrm{a}, \mathrm{b}) \in E_{s t}$ if, and only if, these nodes $(a, b)$ are socially bond to each other. In the proposed approach, the social relation is established based on bonding between two nodes; bonding can be (i) direct bonding or (ii) indirect bonding. When a node has direct or indirect bonding with other nodes, then it is considered as an active node to forward the message.

4.2. Direct Bonding. To explore an efficient link metric that utilizes the nodes bonding relations more accurately to transmit a message to the destination node this proposed approach has examined the following human behavioral features of close bonding by adapting average detachment period. An analysis says that the social meeting pattern in the temporal dynamic graph is undoubtedly distinct from that in the aggregated static chart $[19,20]$. For that reason, this section concentrates on formulating the social meeting patterns from the temporal aspect. In SON, nodes in the network ordinarily have the insight of their prior contact knowledge with other nodes, also termed contact history, e.g., the inter-contact time, contact duration, and detachment period. The average detachment period encloses both the frequency and duration period reflects a strong 
direct bonding relationship with neighbor nodes [3]. In the meantime, the variance of the average detachment period is also listed to mirror the inconsistency in the direct bonding relationship. The deviation in the average detachment period should not neglect. If two conditions have an equal average detachment period, the one along more significant differences would be less preferred since the node would be more uncertain concerning the estimated future detachment period. Thus, this is also necessary to measure the variance of the detachment period dispersion to mirror the deviation using inconsistency metric $V A_{(a, b)}$. The use of average detachment period is more precise to characterize the neighboring relationship rather than only utilizing the inter-contact time or contact duration. Distinctly, a shorter average detachment reflects the consistency in the relationship. A single metric called direct bonding can then deduce from the average detachment period and the variance of the average detachment period. This metric illustrates the relationship among each couple of nodes in SONs and captures the crux of temporal and spatial contact information. So with that, a node-link carrying smaller detachment period and variance show a strong direct bonding to send a message from the source to destination. We denote $D_{(a, b)}$ as the direct bonding between any node a and b with the average detachment period $D P_{(a, b)}$ between two nodes a and $\mathrm{b}$ in a particular time window, $V A_{(a, b)}$ as the variance of the average detachment period between nodes $a$ and $b$ in a specific window of time.

$$
\begin{gathered}
D P_{(a, b)}=\frac{\sum_{i} D P_{i(a, b)}}{n_{a b}} \\
V A_{(a, b)}=V A\left(D P_{i(a, b)}\right)=\left(D P_{(a, b)}-D P_{i(a, b)}\right)^{2} \\
D_{(a, b)}=\frac{1}{D P_{(a, b)}+V A_{(a, b)}}
\end{gathered}
$$

where $D P_{i(a, b)}$ denotes the detachment period between any two nodes a and $\mathrm{b}$ in the $i_{t h}$ encounter, $n_{a b}$ serves as the number of times that $\mathrm{a}$ and $\mathrm{b}$ are aside from each other. The smaller value of $D P_{(a, b)}$ expresses shorter communication suspension between $a$ and $b$. The variance of each detachment period between two nodes is measured by subtracting each time nodes encounter in the given time window and the average, and taking the square.

4.3. Indirect Bonding. Every node can calculate its links close bonding with other neighbor nodes against bonding metric. Though, nodes those are not sharing direct bonding can also share indirect bonding. It has recognized that the power of indirectly associated nodes extremely builds upon the number of different direct or indirect paths connecting them. Accordingly, we examine the influence of numerous implicit $\mathrm{n}$ hop paths in our interpretation of the indirect bonding. Typically, social ties between individuals are asymmetrically reciprocal. Accordingly, to measure the indirect bonding with the help of the social power of various implicit $n$ hop paths between user a and b, this bonding approach considers two hop of social relationship $(n=2)$, where $\mathrm{n}$ shows the length of all possible implicit two-hop paths between a and b. Here a social graph is considered which connects nodes with links weighted based on the power of their direct social bonding communications. For this scenario we are assuming $P_{a, b}$ as the group of divergent implicit paths carrying length $\mathrm{n}$ and accompanying indirect bonding between nodes a and b, $D_{(a, c)}$ and $D_{(c, b)}$ is the direct bonding weight of node a, c and b, c. $N_{a}$ is the neighbor set of node a. Notation $\mathrm{c} \in N_{a}$ is defined as the social power between node a and node b from node a viewpoint over the implicit path of n-hop as:

$$
I_{(a, b)}=1-\prod_{c \in N_{a}} 1-\frac{D_{(a, c)} \times D_{(c, b)}}{2}
$$

4.4. Forwarding Strategy. This section illustrates the Bonding based forwarding technique summarized in the first algorithm. This forwarding technique imitates the message exchange between nodes $a$ and $b$. Beginning with the encounter of a message $x$ node $a$ calculates the direct bonding with as neighbor nodes by measuring nodes average detachment period and variance. After figuring the direct bonding of neighboring nodes, the direct bonding weight assigns to node a neighbors links. Subsequently, those nodes are selected for 
forwarding among $n$ neighboring nodes as a contender for next hop whose are carrying direct bonding weight values more significant than the bonding weight of current node with destination $b$ (e.g., node as neighbor nodes $N_{i}$ ). Select those nodes from $n$ neighboring nodes as a candidate for next hop whose value of bonding weights $\left(D_{(a, b)}<D_{\left(N_{i}, b\right)}\right)$. Let this set of nodes be $L$.

For indirect forwarding, first, indirect bonding is calculated by using implicit $n$ hop paths knowledge between node $a$ and $b$. The extension algorithm observes two steps:

1. Now each node $a$, find bonding node $k$ carrying weakest direct bonding weight such that $D_{(a, b)}=$ $\min \left[D_{(k, b)}\right]$ where $k \in N_{i}$. This weakest normalized direct bonding weight refer to as $\rho_{a}$.

2. For each $m$ of as n-hop implicit nodes, if $I_{(m, b)} \geq \rho_{a}$, the node $m$ is infused in the candidate peer set of node $a$. Instinctively, this guarantees that the social power between $a$ and $b$, positioned at length $n$ in the social opportunistic network graph, is at least as strong as node as the weakest direct bonded node. Let this set of nodes be $M$.

Now take the nodes belonging to the intersection of sets $L$ and $M$ and then forwards the message copy to these nodes.

5. Algorithms and Sub-Routines of Bonding based Forwarding Approach. In this section, the algorithm and sub-routines used in BBFT algorithm for sending the message from the source to the destination are discussed in detail. Algorithm 1, Algorithm 2 and Algorithm 3 are mentioned below.

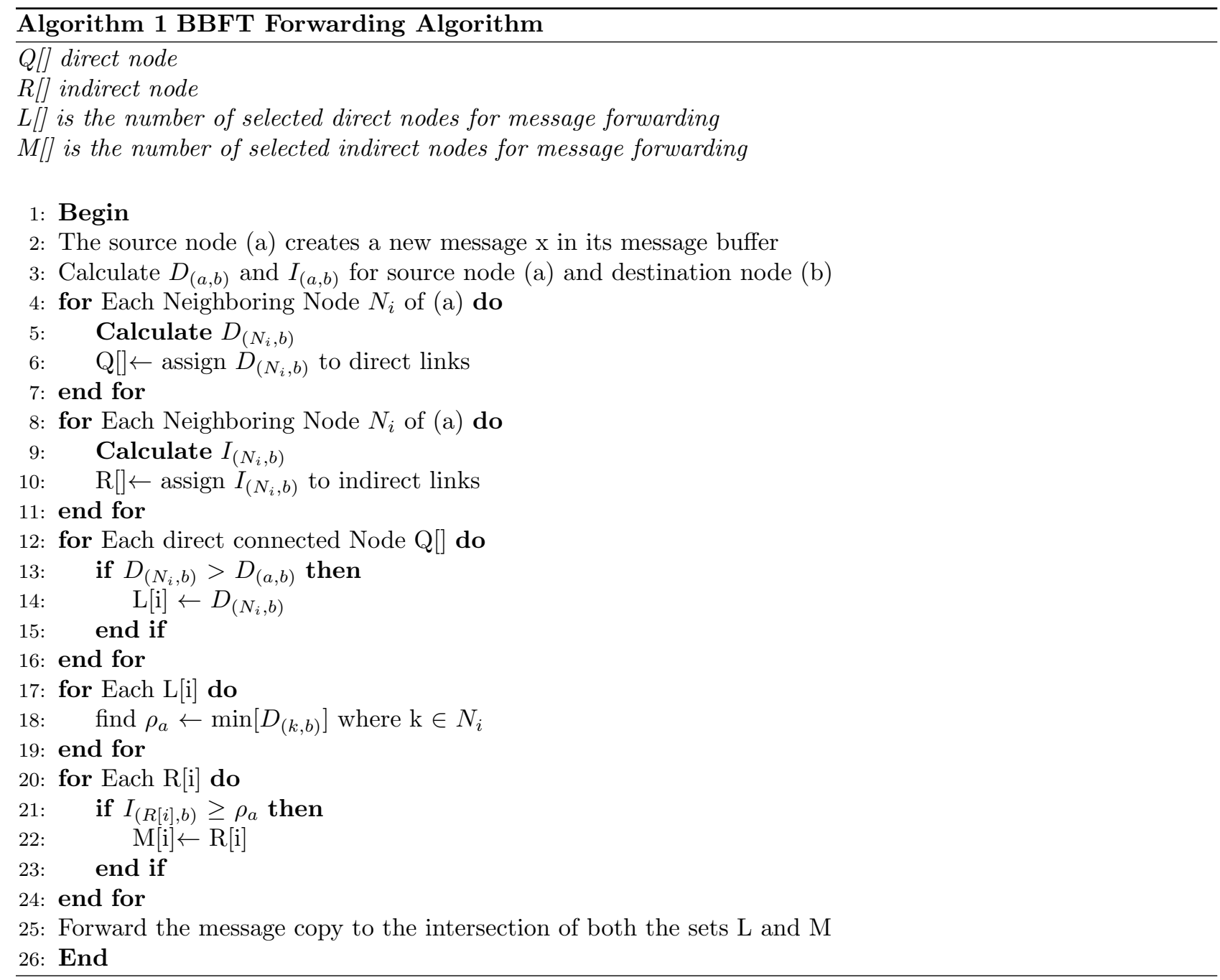



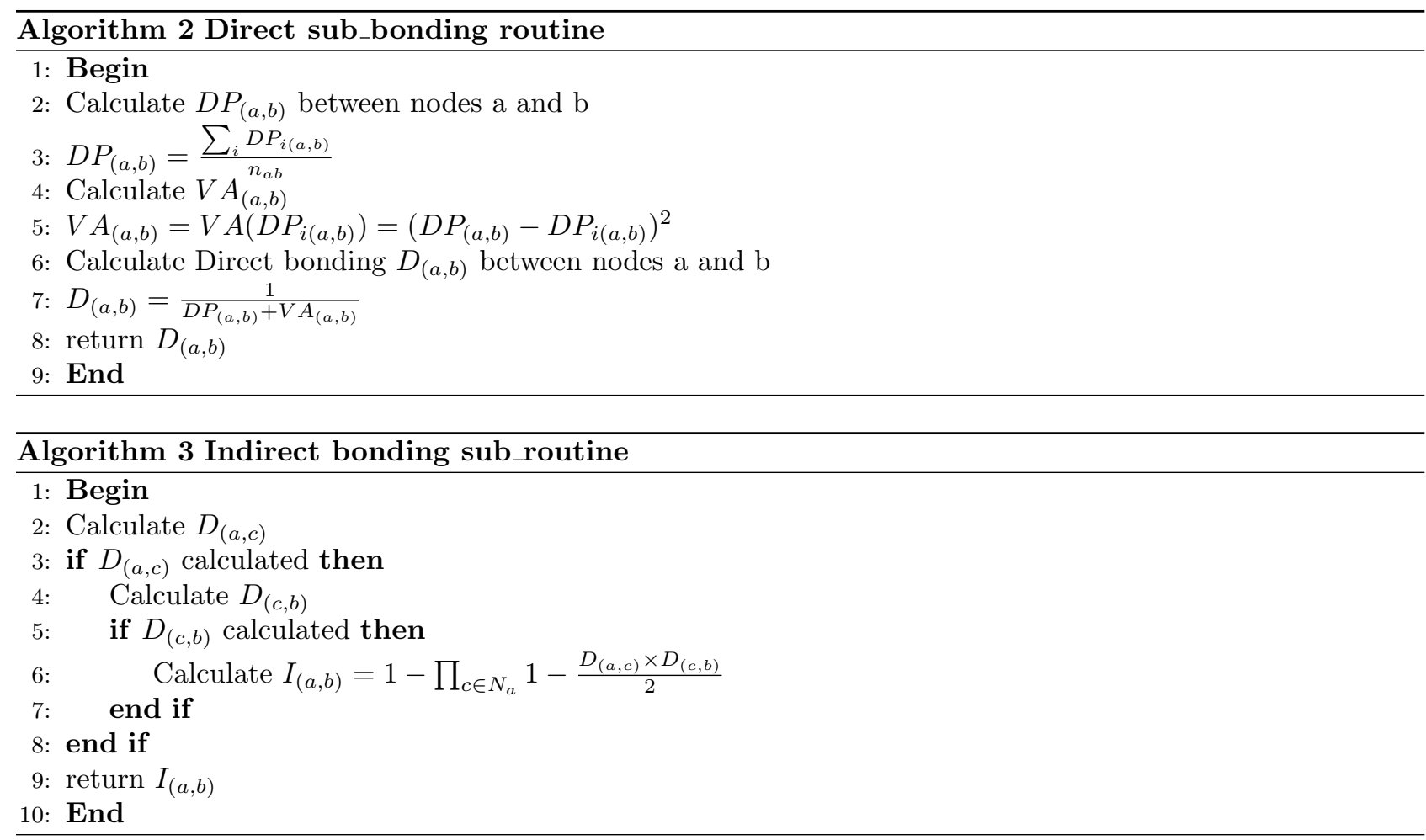

\section{Simulation Setup and Results.}

\subsection{Data Forwarding Experiment.}

Algorithm Comparison. In this segment, the performance of the Bonding based forwarding technique has compared against few encounter based routing protocols (Epidemic [4], PROPHET [5], BUBBLE RAP [8], Interaction based routing protocol [2]). Distinctly, the last two have social-aware properties further.

Simulation Setup. The ONE simulator [21] has chosen as a performance evaluation tool for proposed Bonding based forwarding technique. The default framework for the simulation arranged as described in Table 6.1 and Table 6.2. These establish the standard configuration for all the four forwarding protocols Epidemic, Prophet, BubbleRap, and Interaction Based.

The mobile node is partitioned into six groups and out of which two groups serve as pedestrians. The pedestrians belong to first and third group nodes. This pedestrian group form of 40 host nodes each one have carried a buffer size of $5 \mathrm{MB}$ and motion according to the movement model named as Shortest path map based movement model. The nodes of the pedestrian groups follow a walking speed of $0.5-1.5 \mathrm{~m} / \mathrm{s}$. The second group also have 40 nodes, but these nodes depict car. The speed of the car nodes are between 2.7-13.9 m/s, buffer size and movement model followed by car group same as pedestrian groups.

The rest three groups form of two nodes each depicting tram nodes. The tram group nodes motion according to the Route based map movement model, have a buffer space of $50 \mathrm{MB}$ and a speed of $710 \mathrm{~m} / \mathrm{s}$. A word size of $4500 \times 3400 \mathrm{~m}^{2}$ has defined to the movement models. The mobile nodes of all six groups have a transmission range of $10 \mathrm{~m}$ and the transmission speed of $2 \mathrm{Mbps}$. The communication is initiated between nodes by two distinct interfaces that have detailed in Table 6.2. The pedestrian and car groups adapt Bluetooth interface for communication, at the same time tram groups adopt the high-speed interface. Each message produces in the Social opportunistic network has credited value of $300 \mathrm{sec}$ as a Time-to-live (Message TTL). In the system, messages generated at regular intervals of 25-35 sec and these messages have a message size between $500 \mathrm{~KB}$ to $1 \mathrm{MB}$. The time of simulation is assigned each of the five protocols (Bonding based, Epidemic, Prophet, BubbleRap, and Interaction Based) is 43200 sec. 
Table 6.1: Common default settings of simulation specification.

\begin{tabular}{|c|c|}
\hline Specification & Value \\
\hline Simulation area & $4500 \mathrm{~m} * 3400 \mathrm{~m}$ \\
\hline Simulation time & 43200 \\
\hline Total no. of nodes & 126 \\
\hline Total No. of node groups & 6 \\
\hline Speed range & $0.5-1.5 \mathrm{~m} / \mathrm{s}$ \\
\hline Total no. of movement models & 2 \\
\hline Message TTL & $300 \mathrm{~s}$ \\
\hline Buffer size & $5 \mathrm{M}$ \\
\hline Message size & $500 k, 1 \mathrm{M}$ \\
\hline Transmission speed & $250 \mathrm{k}$ \\
\hline Transmission range & 10 \\
\hline
\end{tabular}

Table 6.2: Group Specific default settings of simulation specification.

\begin{tabular}{|c|c|c|c|}
\hline Specification & Pedestrian & Car & Tram \\
\hline No. of groups & 2 & 1 & 3 \\
\hline Nodes in each group & 80 & 40 & $50 M$ \\
\hline Node buffer capacity & $5 M$ & $5 M$ & $7-10 \mathrm{~m} / \mathrm{s}$ \\
\hline Speed range & $0.5-1.5 \mathrm{~m} / \mathrm{s}$ & $2.7-13.9 \mathrm{~m} / \mathrm{s}$ & Map Route Based \\
\hline Movement Model & Shortest path & Map Route Based & Bluetooth, High Speed \\
\hline Interface & Bluetooth & Bluetooth & Bigh
\end{tabular}

The specified parameters of the network have been fluctuated to produce results for comparison:

1. fluctuating the buffer size (MB): The buffer size in the simulation are assorted as 5, 10, 15, 20, 25 and 30 to observe the performance of Bonding based forwarding technique protocol.

2. fluctuating the Time-to-live(Sec): The TTL in the simulation are assorted as 100, 200, 300, 400, 500.

3. fluctuating the message size: The message size in the simulation assort as (100KB to 200KB), (200KB to $300 \mathrm{~KB}),(300 \mathrm{~KB}$ to $400 \mathrm{~KB}),(500 \mathrm{~KB}$ to $1 \mathrm{MB})$ and $(1 \mathrm{MB}$ to $2 \mathrm{MB})$.

4. fluctuating the number of nodes in the simulation: The total no of nodes in the simulation are assorted as $20,40,80,100$ and 120 .

5. fluctuating the number of nodes in the simulation: The total no of nodes in the simulation are assorted as $20,40,80,100$ and 120 .

The performance metrics used are:

1. Message delivery probability: the Delivery probability of the nodes implies the estimate of successful acceptance of messages by their destination. Delivery probability is supposed to be high in the course of the simulation.

2. Overhead ratio: Overhead ratio is the average number of forwarded replicas per message. Network resource utilization and bandwidth efficiency evaluate by overhead ratio. An efficient routing protocol causes a small overhead ratio.

3. Dropped messages: Dropped messages show the total number of words descended from the node buffers. Every node contains a limited buffer space in the network, and an excellent routing protocol attempts to lower its utilization.

4. Average latency: It is the average of the difference between the message delivery time and message creation time.

6.2. Comparing the performance of three protocols at varying the buffer size. Figure 6.1 a- $d$ understands the outcome of the increase in buffer size by performance metrics. The performance of BBFT compare to Interaction based, BubbleRap, Prophet, and Epidemic and it can recognize from the Fig.6.1a that BBFT has a greater no. of message delivery as compare to protocols as mentioned earlier. It has interpreted from Fig.6.1a that fluctuating the buffer size of the nodes in the increasing order results in the increase of 


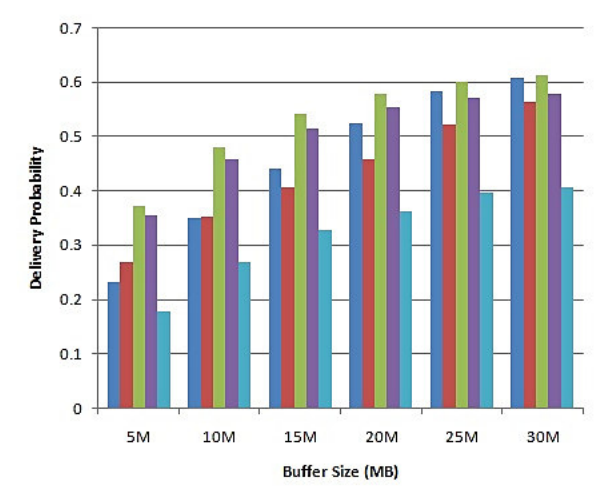

(a) Buffer size vs. delivery probability

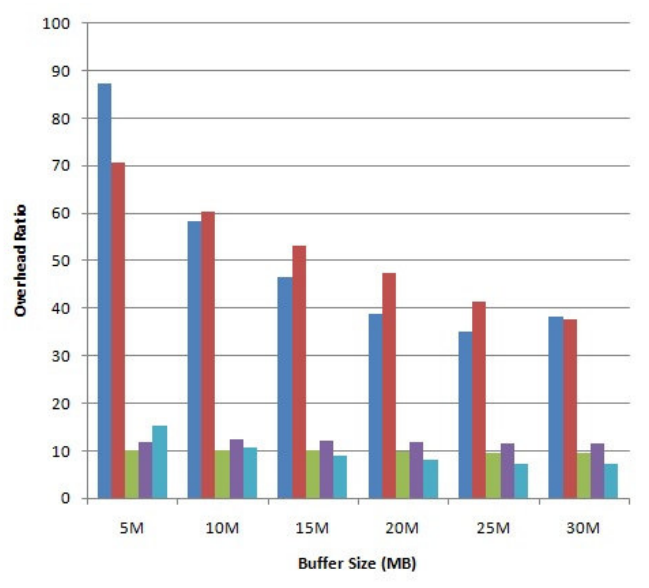

(c) Buffer size vs. Overhead ratio

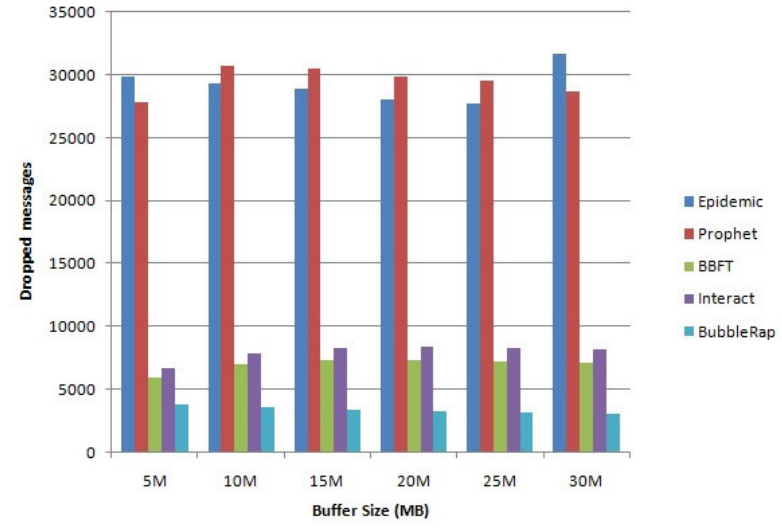

(b) Buffer size vs. Dropped messages

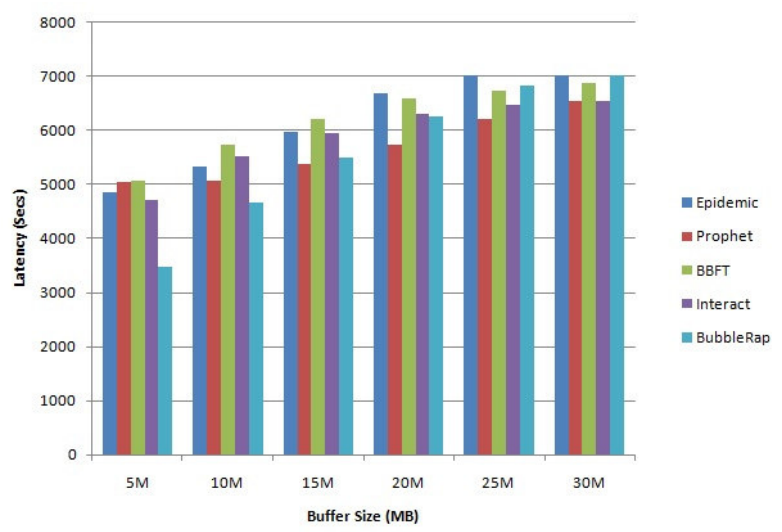

(d) Buffer size vs. Average latency

Fig. 6.1: Performance Metrics versus Buffer size

delivery probability. As the buffer size increase from $5 \mathrm{Mb}$ to $30 \mathrm{Mb}$, the capacity to hold the messages enhance by the nodes that increase messages forwarded to the destination. Figure $6.1 \mathrm{~b}$ shows that as the buffer size of nodes increases the number of dropped messages also mostly increases. This above happens because, with the increase of buffer size, no significant improvement observe as the buffer has reached the saturation. That is, messages have already been delivered to the destination before the buffer gets filled and they need to drop. The number of dropped messages of BBFT is far less than Interaction based, Prophet and Epidemic and a little bit more than BubbleRap. Figure 6.1c interprets that the overhead ratio drops with the rise in the buffer size and it is lesser than Epidemic, Prophet, and Interaction based routing protocols. In the process of sending messages, the increase in buffer size results decreases in overhead ratio when a source node has more competent neighbor nodes. The reason behind this overhead ratio drop in BBFT is the appropriate selection of next hop hosts by exploiting the bonding metric. It can be interpreted from Fig.6.1d that the average latency increments with the gain in buffer size. As the buffer size increases the message drop decreases due to that more message will grab the opportunity to be conveyed to the destination. As a result of that average latency is increased. BBFT has increased average latency as compare to Epidemic, Prophet, BubbleRap, and Interaction based routing protocols.

The BBFT is $16.36 \%$ better Epidemic, $24.12 \%$ better than Prophet, $66.51 \%$ better than BubbleRap and 


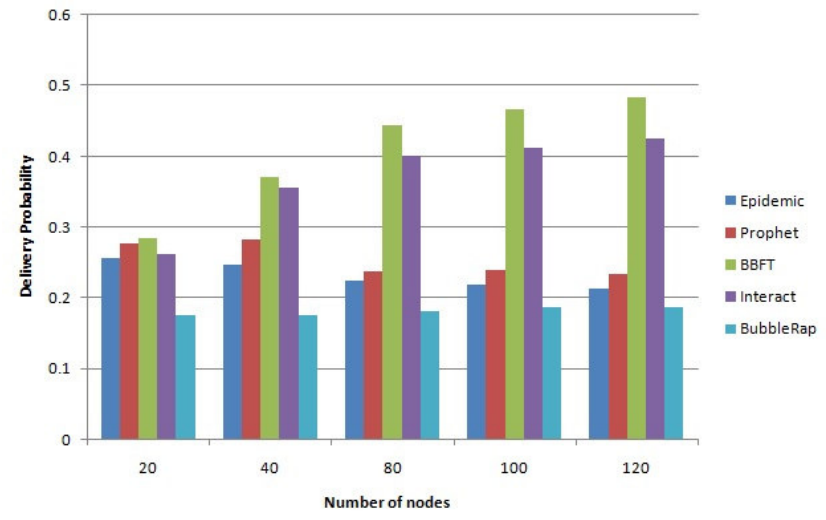

(a) Number of nodes vs. delivery probability

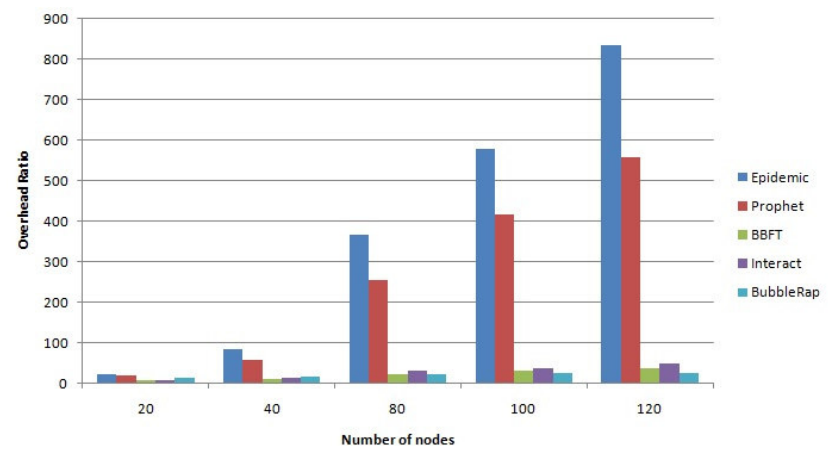

(c) Number of nodes vs. Overhead ratio

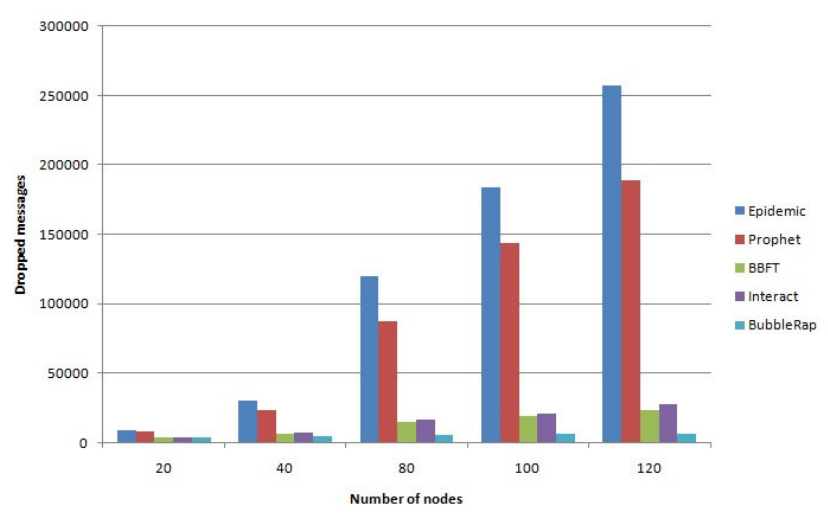

(b) Number of nodes vs. Dropped messages

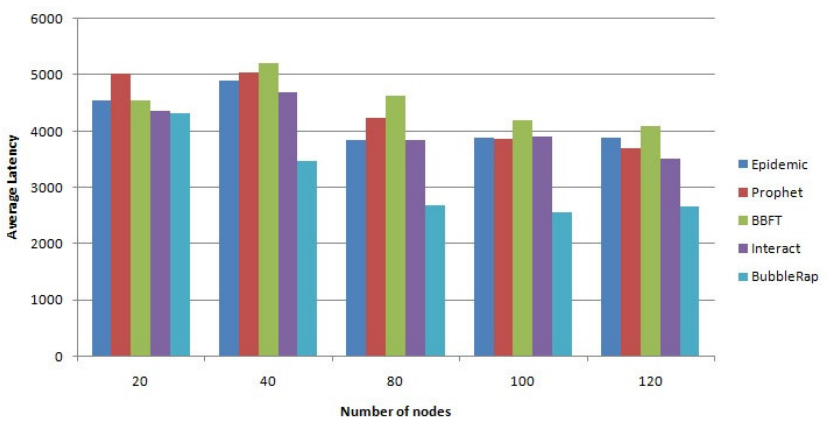

(d) Number of nodes vs. Average latency

Fig. 6.2: Performance Metrics versus Total number of nodes

$5.06 \%$ better than Interaction based regarding the number of messages delivered. The number of messages drops in BBFT is decreased which is 8437 whereas Epidemic, Prophet, and Interaction based has 26667, 35438, 9588. The BubbleRap has 4110 drop which is slightly better. The overhead ratio of BBFT is $80.59 \%$ decreased than Epidemic, 80.96\% declined than Prophet and 16.84\% decreased than Interaction based. The Bubblerap has $3.78 \%$ improved overhead ration as compare to BBFT. The average latency of BBFT is $0.85 \%$ more than Epidemic, 9.45\% more than Prophet, $4.78 \%$ more than Interaction based and 10.25\% more than Bubblerap.

6.2.1. Comparing the performance of three protocols at different number of nodes. Figure 6.2 a-d interprets the aftereffect of increase in the number of nodes in the network by performance metrics. The performance of BBFT compares to Interaction based, BubbleRap, Prophet, and Epidemic. It can visualize from Fig.6.2a, by varying the number of nodes from 100 to 500, the average delivery probably for BBFT increases as compared to Epidemic, Prophet, BubbleRap and Interaction based protocols. The message delivery probability for BBFT hikes as more hosts is joined, which can associate with the corresponding gain in the number of message transmission in the network. It can be inspected in Fig.6.2b, as the number of hosts increases in the network, the number of dropped messages are decline. This reaction is due to the hike in the number of message transmission and is in interrelation with huge delivery probability examined for message delivery. The no. of dropped messages for BBFT decrease as compare to Epidemic, Prophet, and Interaction based protocols. By scanning Fig.6.2c, it observes that by changing the number of nodes, the overhead ratio of messages for BBFT decrease as compared to Epidemic, Prophet, and Interaction based protocols. The minimum overhead ratio of BBFT exploits more convoluted information from the network parameters and then process it to compose better 


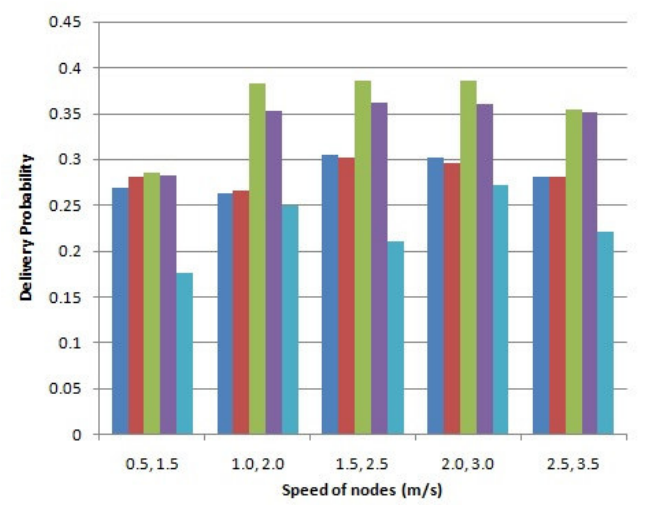

(a) Speed of nodes vs. delivery probability

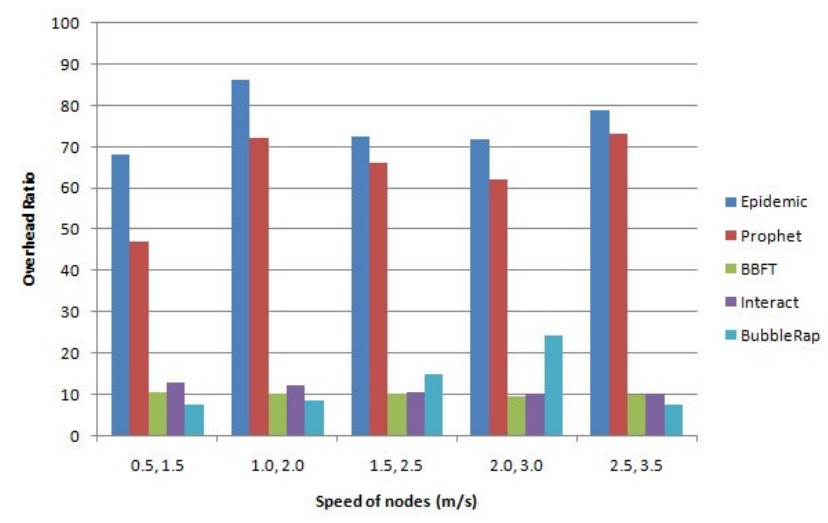

(c) Speed of nodes vs. Overhead ratio

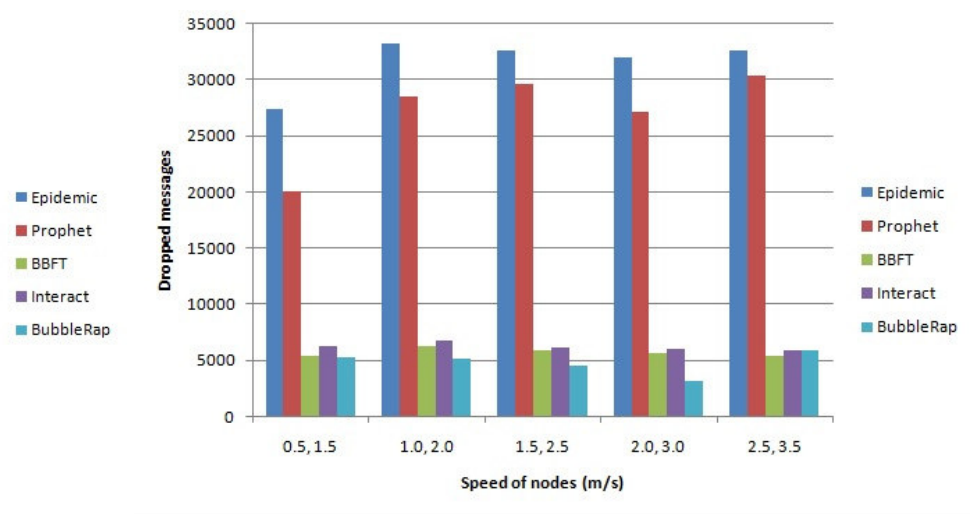

(b) Speed of nodes vs. Dropped messages

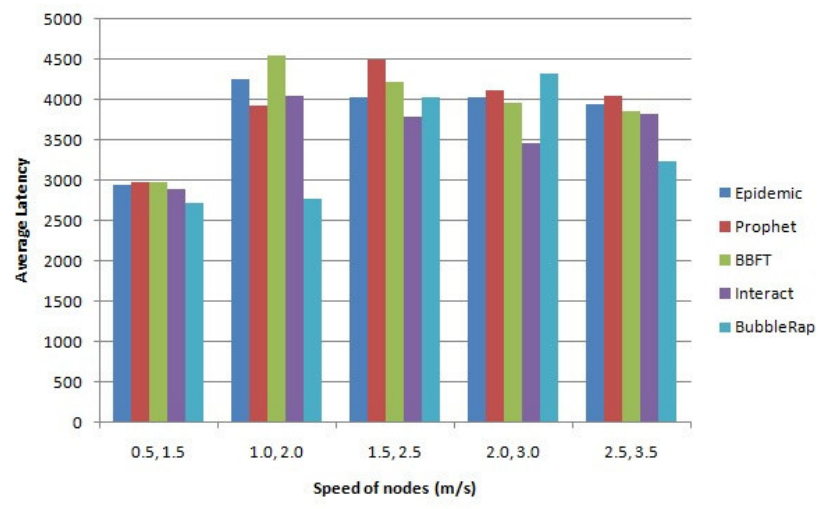

(d) Speed of nodes vs. Average latency

Fig. 6.3: Performance Metrics versus Speed of nodes

decisions about next hop choice which lowers the number of message replicas in the network. It reduces network resource utilization and thus cutting down the overhead ratio. It can interpret from Fig.6.2d that in BBFT the average latency hikes with increment in the number of messages. The reason is that as the nodes raise, the communications a sender node can have with its neighbor nodes also rises. Due to that BBFT uses some time in deciding to choose the best possible node as next hop for conveying the message. BBFT has increased average latency as compare to Epidemic, Prophet, BubbleRap, and Interaction based routing protocols.

The BBFT is $77.3 \%$ better Epidemic, $61.71 \%$ better than Prophet, $126.5 \%$ better than BubbleRap and $10.47 \%$ better than Interaction based regarding the number of messages delivered. The number of messages dropped in BBFT decrease remarkable which is 13091 whereas Epidemic, Prophet, and Interaction based have 119961, 90176, 14981. The BubbleRap has 4798 drop which is better. The overhead ratio of BBFT is $94.69 \%$ decreased than Epidemic, 92.29\% declined than Prophet and 21.58\% decreased than Interaction based. The BubbleRap has $4.8 \%$ improved overhead ration as compare to BBFT. The average latency of BBFT is $7.61 \%$ more than Epidemic, 3.82\% more than Prophet, $11.7 \%$ more than Interaction based and $44.38 \%$ more than BubbleRap.

6.2.2. Comparing the performance of three protocols at different speeds. The performance of the distinct protocols observe as a parameter of mobility, the range of speed of the 80 nodes in the two pedestrian groups (as per the baseline settings) is varied from $0.51 .5 \mathrm{~m} / \mathrm{s}$ to $2.53 .5 \mathrm{~m} / \mathrm{s}$. The outcome of the delivery probability, the number of dropped messages, overhead ratio, and average latency, has been illustrated in Fig.6.3 
ad accordingly. By varying the speed of the nodes in the increasing order results gain in delivery probability of the messages also. As this scene from Fig.6.3a that that BBFT has a leading no. of message delivery in contrast to the Interaction based, BubbleRap, Prophet and Epidemic protocols. Figure 6.3b interprets that when messages are transmitted further into the network along with an expansion in mobility of the hosts, this results to a higher opportunity of the message reaching nearer to its destination host in the network. The number of dropped messages for BBFT increments randomly with the increase in speed of the nodes, as message propagation raises with the escalation in the node mobility which results in more number of dropped messages. Changing the speed of the nodes leads in a small decrease in the overhead ratio for BBFT which validate as the message transmissions in the network increase Fig.6.3c. It can interpret from Fig.6.3d that in BBFT the average latency hikes randomly with the increase in speed of nodes. BBFT has increased average latency as compare to Epidemic, Prophet, BubbleRap, and Interaction based routing protocols.

The BBFT is $26.42 \%$ better than Epidemic, $25.59 \%$ better than Prophet, $59.15 \%$ better than BubbleRap and $4.95 \%$ better than Interaction based regarding the number of messages delivered. The number of messages dropped in BBFT is decrease remarkable which is 5678 whereas Epidemic, Prophet, and Interaction based has 31648,27145 , and 6162. The BubbleRap has 4753 drop which is slightly better. The overhead ratio of BBFT is $86.82 \%$ decreased than Epidemic, $84.49 \%$ declined than Prophet, and $10.11 \%$ decreased than Interaction based and $19.9 \%$ decreased than the BubbleRap. The average latency of BBFT is $1.9 \%$ more than Epidemic, $0.04 \%$ more than Prophet, $8.57 \%$ more than Interaction based and 14.70\% more than BubbleRap.

6.2.3. Comparing performance of three protocols at Varying the Message Size. Figure 6.4 a-d interprets the outcome of the increase in message size by performance metrics. The performance of BBFT compare to Interaction based, BubbleRap, Prophet, and Epidemic and it can be recognized from Fig.6.4a that BBFT has larger no. of messages delivered as compared to protocols as mentioned above. It interprets from Fig.6.4a that fluctuating the message size of the nodes in the increasing order results in a decrease of delivery probability. The message sizes increase from 100k-200k to 1M-2M, the node buffer capacity to hold the messages decrease which results in the reduction of messages forwarded to the destination. Figure 6.4b shows that as the message size of nodes increases the number of dropped messages also mostly decreases. The BBFT has lesser number of lost messages than Interaction based, Prophet and Epidemic and a little bit more than BubbleRap. Figure 6.4c shows that the overhead ratio of BBFT decreases with increase in the message size and is less than PRoPHET. Figure6.4d Shows that the average latency increases with the increase in message size. This interpretation is because, with increased message size, number of messages are dropped from it reduces. Thus, more words get the chance to be delivered to the destination which increases the average latency.

The BBFT is $21.06 \%$ better than Epidemic, $20.76 \%$ better than PRoPHET, $3.02 \%$ better than INTERACT and $22.98 \%$ better than BubbleRap regarding Delivery Probability. The number of messages dropped in BBFT is decrease remarkable which is 13514 whereas Epidemic, Prophet, and Interaction based have 84736, 68648, and 14784. The BubbleRap has 4611 drop which is better. The overhead ratio of BBFT is $87.02 \%$ less than Epidemic, 83.91\% less than PRoPHET, $12.94 \%$ less than INTERACT and $11.48 \%$ more than BubbleRap. The latency is $6.40 \%$ more than Epidemic, $4.11 \%$ less than PRoPHET, $4.95 \%$ more than INTERACT and $22.86 \%$ more than BubbleRap.

6.2.4. Comparing performance of three protocols at Varying the Time to Live(TTL). Figure 6.5 a-d interprets the outcome of the increase in TTL by performance metrics. The performance of BBFT compare to Interaction based, BubbleRap, Prophet, and Epidemic and it can recognize from the Fig.6.5a that BBFT has a high delivery ratio as compare to protocols as mentioned earlier. It has interpreted from Fig.6.5a that changing the TTL of the message in the increasing order results in the increase of delivery probability. As the TTL increase from 100 to 500, messages are getting enough time to live in the network to reach the destination. Figure $6.5 \mathrm{~b}$ shows that as the TTL of messages increases the dropping varies a little only. It happens because, with the increase in TTL, no significant improvement observe as we are following the Drop Oldest strategy. The number of dropped messages of BBFT is far less than Interaction based, Prophet and Epidemic and a little bit more than BubbleRap. Figure $6.5 \mathrm{c}$ shows that the overhead ratio decreases slightly with the increase in the TTL. However, the overhead ratio of BBFT remains much less than the Interaction based, BubbleRap, Prophet and Epidemic protocols because of its efficient selection of next hop nodes using the Bonding Metric. It can observe from Fig.6.5d that the average latency increases with increase in TTL. This 


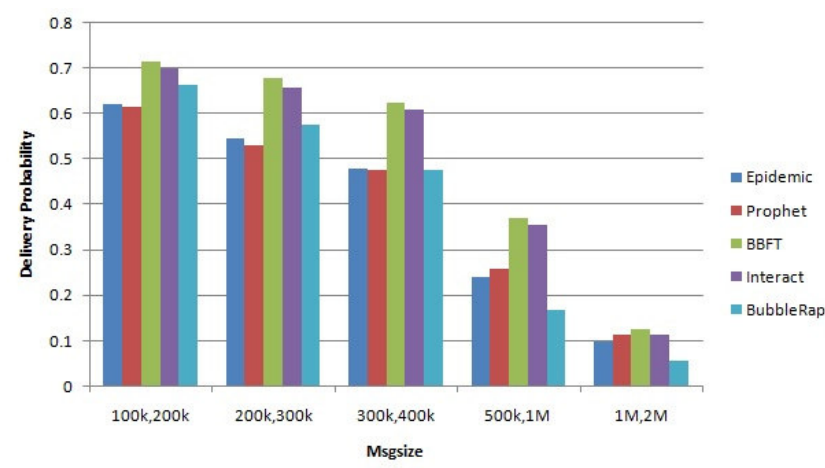

(a) Msgsize vs. delivery probability

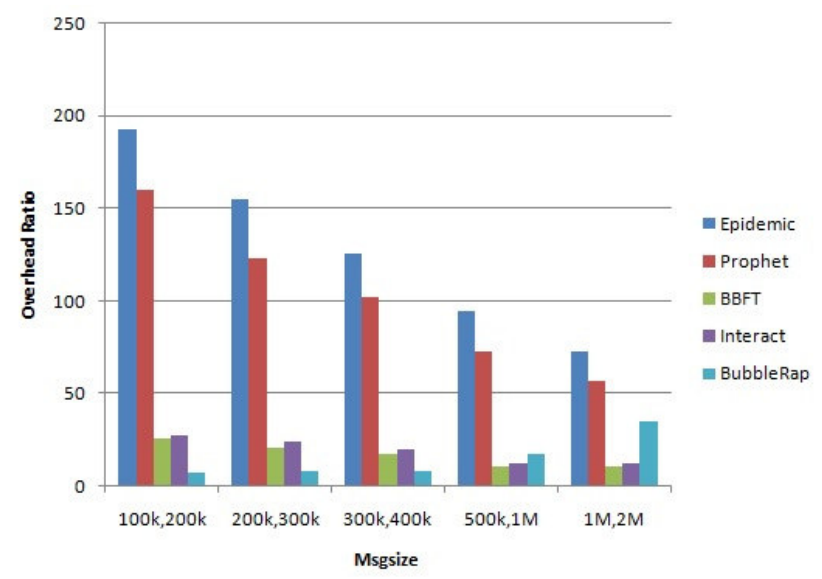

(c) Msgsize vs. Overhead ratio

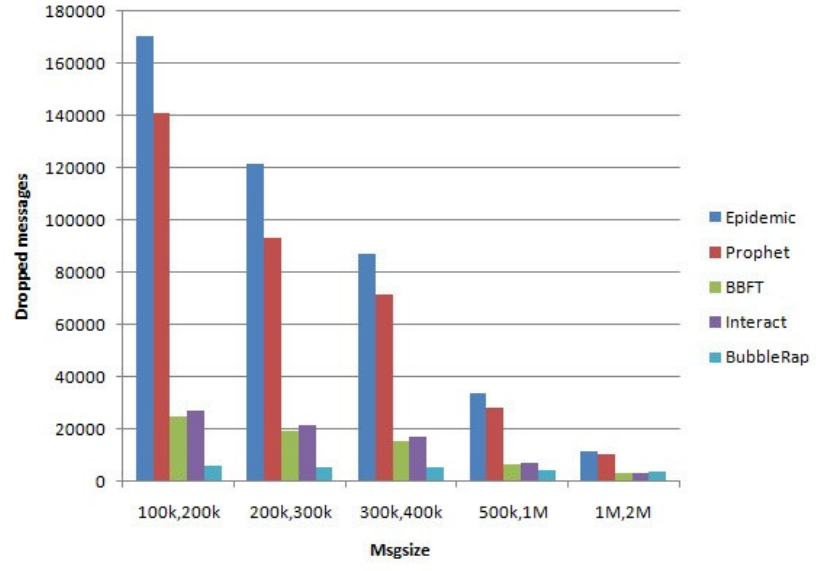

(b) Msgsize vs. Dropped messages

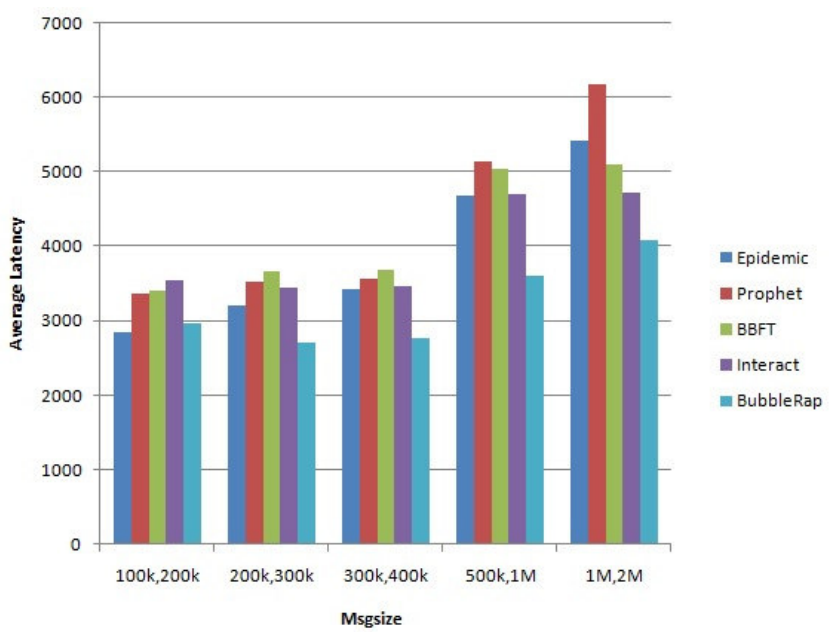

(d) Msgsize vs. Average latency

Fig. 6.4: Performance Metrics versus Msgsize

observation is because with increased TTL lesser number of messages are dropped from it. Thus, more words will get the chance to be delivered to the destination which results in increased average latency.

The BBFT is $31.03 \%$ better than Epidemic, $25.91 \%$ better than PRoPHET, $5.26 \%$ better than INTERACT and $51.72 \%$ better than BubbleRap regarding the number of messages delivered. The number of messages dropped in BBFT decrease remarkable which is 5821 whereas Epidemic, Prophet, and Interaction based have 32617, 26651, and 6616. The BubbleRap has 3952 drop which is better. The overhead ratio of BBFT is 817.39\% less than Epidemic, 591.59\% less than PRoPHET, 21.74\% less than INTERACT and 56.93\% less than BubbleRap. The average latency of BBFT is $9.81 \%$ more than Epidemic, 3.02\% more than PRoPHET, $10.30 \%$ more than INTERACT and $31.90 \%$ more than BubbleRap.

7. Conclusion. In this attempt, a novel routing algorithm BBFT propose that evaluates the strength of direct and indirect bonding and avail it to extend the preferred set of nodes in the Social Opportunistic Network while preserving the social patterns that are the most desirable in SON. Specifically, by taking into account both the intensity of the number of encounters and the number of associated paths results in an average 


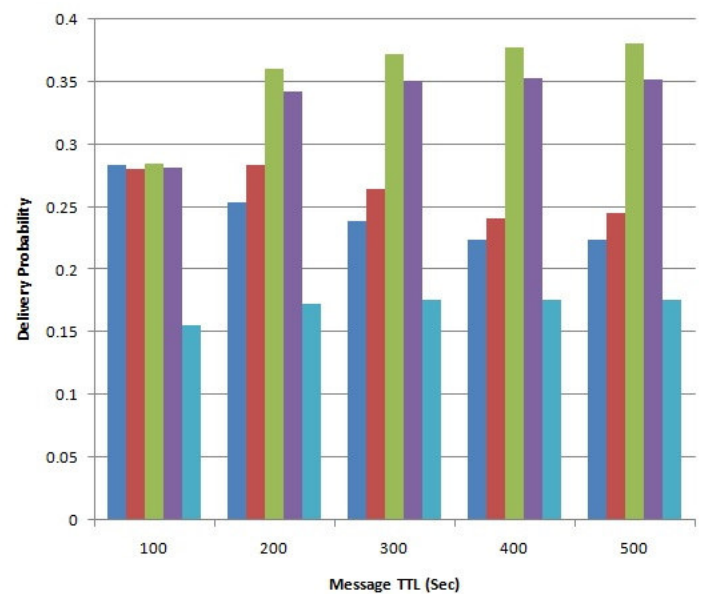

(a) Message TTL vs. delivery probability

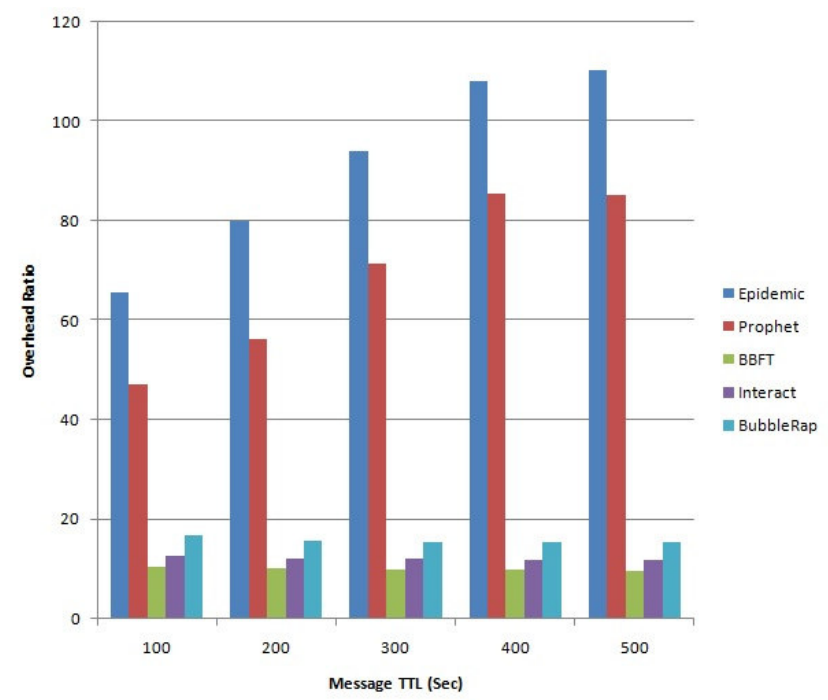

(c) Message TTL vs. Overhead ratio

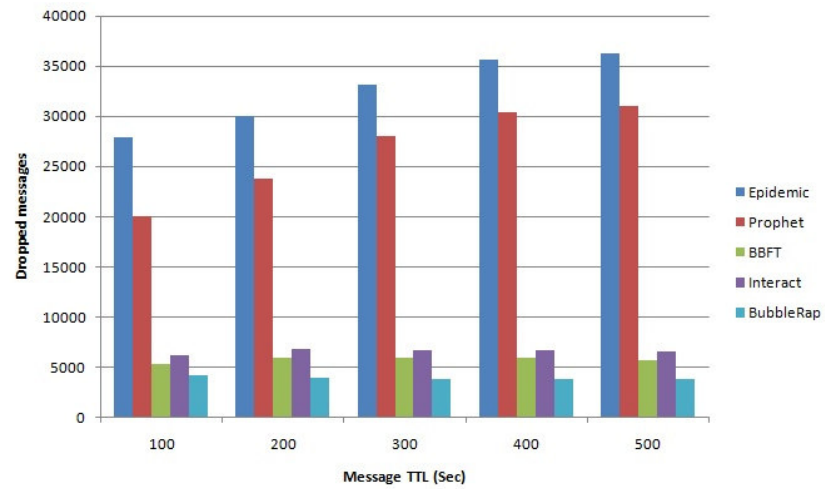

(b) Message TTL vs. Dropped messages

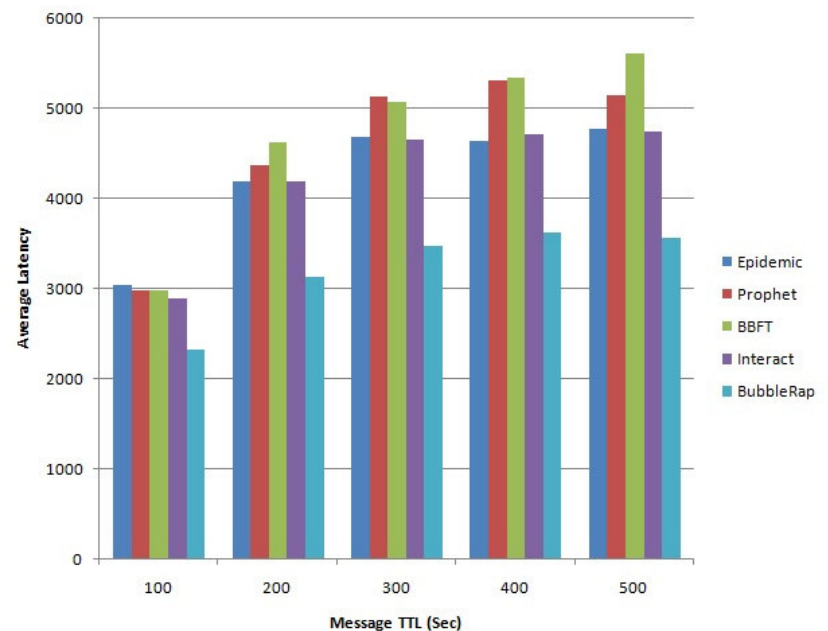

(d) Message TTL vs. Average latency

Fig. 6.5: Performance Metrics versus Message TTL

detachment period and variance, the preferred set of nodes extend. The enlarged node set incorporate not only the directly connected nodes in the SONs but also companion nodes located two hops distant with whom node has an indirect bonding higher than that of nodes weakest directly bonded node. The BBFT interpret against Epidemic, PRoPHET, BubbleRap, and Interaction based routing protocols. It functions significantly fine concerning the delivery probability of the nodes, as a result of selecting improved and dependable next hop nodes. The overhead ratio and dropped messages for BBFT are also lower than the other protocols. In future, the BBFT can interpret with more routing protocols like HiBOp and CAR. Average latency is one parameter in which other protocols still behave better than BBFT and hence enhancing the average message latency while maintaining the substantial delivery probability of BBFT is another field of future work. Future efforts will be executed to accomplish the BBFT protocol secure, and the issue of energy consumption also taken into account. 


\section{REFERENCES}

[1] D. J. Brass, K. D. Butterfield, And B. C. Skaggs, "Relationships and unethical behavior: A social network perspective," Academy of management review, vol. 23, no. 1, pp. 14-31, 1998.

[2] Y. Li AND R. BARTos, "Interaction based routing algorithm for opportunistic mobile social networks," in Consumer Communications \& Networking Conference (CCNC), 2017 14th IEEE Annual. IEEE, 2017, pp. $492-497$.

[3] F. Li AND J. WU, "Localcom: A community-based epidemic forwarding scheme in disruption-tolerant networks." in $S E C O N$, vol. 9, 2009, pp. 574-582.

[4] A. Vahdat, D. Becker et al., "Epidemic routing for partially connected ad hoc networks," 2000.

[5] A. Lindgren, "D0ria a, schelen o," Probabilistic routing in intermittently connected networks, vol. 7 , no. 3, p. 1, 2003.

[6] A. MtibaA, M. May, C. Diot, And M. Ammar, "Peoplerank: Social opportunistic forwarding," in Infocom, 2010 Proceedings IEEE. IEEE, 2010, pp. 1-5.

[7] E. M. DAly AND M. HaAhr, "Social network analysis for routing in disconnected delay-tolerant manets," in Proceedings of the 8th ACM international symposium on Mobile ad hoc networking and computing. ACM, 2007, pp. 32-40.

[8] P. Hui, J. Crowcroft, And E. Yoneki, "Bubble rap: Social-based forwarding in delay-tolerant networks," IEEE Transactions on Mobile Computing, vol. 10, no. 11, pp. 1576-1589, 2011.

[9] T. Spyropoulos, K. Psounis, and C. S. Raghavendra, "Spray and wait: an efficient routing scheme for intermittently connected mobile networks," in Proceedings of the 2005 ACM SIGCOMM workshop on Delay-tolerant networking. ACM, 2005 , pp. 252-259.

[10] J. PARK, J. Lee, S.-K. Kim, K. JAng, And S.-B. YAnG, "A forwarding scheme based on swarm intelligence and percolation centrality in opportunistic networks," Wireless Networks, vol. 22, no. 8, pp. 2511-2521, 2016.

[11] Q. Li, S. ZHU, AND G. CAO, "Routing in socially selfish delay tolerant networks," in INFOCOM, 2010 Proceedings IEEE. IEEE, 2010, pp. 1-9.

[12] S. K. Dhurandher, D. K. Sharma, I. Woungang, And S. Bhati, "Hbpr: history based prediction for routing in infrastructure-less opportunistic networks," in Advanced Information Networking and Applications (AINA), 2013 IEEE 27th International Conference on. IEEE, 2013, pp. 931-936.

[13] A. Chhabra, V. Vashishth, And D. K. Sharma, "A fuzzy logic and game theory based adaptive approach for securing opportunistic networks against black hole attacks," International Journal of Communication Systems, vol. 31, no. 4, p. e3487, 2018.

[14] — "A game theory based secure model against black hole attacks in opportunistic networks," in Information Sciences and Systems (CISS), 2017 51st Annual Conference on. IEEE, 2017, pp. 1-6.

[15] — - "Seir: A stackelberg game based approach for energy-aware and incentivized routing in selfish opportunistic networks," in Information Sciences and Systems (CISS), 2017 51st Annual Conference on. IEEE, 2017, pp. 1-6.

[16] D. K. Sharma, S. K. Dhurandher, M. S. Obaidat, A. Bansal, and A. Gupta, "Genetic algorithm and probability based routing protocol for opportunistic networks," in Computer, Information and Telecommunication Systems (CITS), 2017 International Conference on. IEEE, 2017, pp. 58-62.

[17] D. K. Sharma, S. K. Dhurandher, I. Woungang, A. Bansal, And A. Gupta, "Gd-car: A genetic algorithm based dynamic context aware routing protocol for opportunistic networks," in International Conference on Network-Based Information Systems. Springer, 2017, pp. 611-622.

[18] D. K. Sharma, S. K. Dhurandher, D. Agarwal, and K. Arora, "krop: k-means clustering based routing protocol for opportunistic networks," Journal of Ambient Intelligence and Humanized Computing, pp. 1-18, 2018.

[19] W. GAO, Q. Li, B. ZhaO, AND G. CAO, "Multicasting in delay tolerant networks: a social network perspective," in Proceedings of the tenth ACM international symposium on Mobile ad hoc networking and computing. ACM, 2009, pp. 299-308.

[20] H. Zhou, J. Chen, H. Zhao, W. GaO, and P. Cheng, "On exploiting contact patterns for data forwarding in duty-cycle opportunistic mobile networks," IEEE Transactions on Vehicular Technology, vol. 62, no. 9, pp. 4629-4642, 2013.

[21] A. KerAnen, "Opportunistic network environment simulator," Special Assignment report, Helsinki University of Technology, Department of Communications and Networking, 2008.

Edited by: Khaleel Ahmad

Received: Nov 11, 2018

Accepted: Feb 11, 2019 
